\title{
Use of urine samples as controls for treatment of a Chlamydia trachomatis infection
}

Chlamydia trachomatis is the most common sexually transmitted bacterial pathogen worldwide. Because most infected individuals are asymptomatic, the actual number of reported cases represents only a fraction of the infected population. If left untreated, this silent infection can evolve, in women, into pelvic inflammatory disease or lead to infertility. Since the infection is easily and effectively treatable with antibiotics (e.g. doxycycline and azitromycin), detection and treatment of infected individuals is an important part of chlamydia control programs.

Nucleic acid amplification tests are an important advance in the field of C. trachomatis detection, and are now in routine use for the screening and diagnosis of C. trachomatis infections. These diagnostic assays can be performed with various specimens, including vaginal, endocervical and urethral swabs, and urine specimens. There is some debate, however, whether female urine specimens are sufficiently reliable for an optimal, sensitive diagnosis of a C. trachomatis infection (Van Doornum et al., 2001; Moncada et al., 2003; Gaydos et al., 2004). Urine collection is preferred for practical reasons, because it is non-invasive and much less burdensome for the individuals being tested than the other sample-collection methods. Urine samples might be suitable specimens for the monitoring of treatment efficiency.

Roosendaal et al. (1993) and Vogels et al. (1993) demonstrated that C. trachomatis DNA could be found in smears for up to 3 weeks after antibiotic treatment; this was probably caused by the presence of residual DNA or DNA from non-viable C. trachomatis organisms. In urine samples, Claas et al. (1991) and Bianchi et al. (1998) reported the clearance of $C$. trachomatis within 6 or 7 days post-therapy. However, there is no 'gold standard' for successful therapy.

The purpose of the present study was to evaluate whether a PCR assay for $C$. trachomatis in urine samples is a useful tool to study the response to treatment of a chlamydia infection. We asked general practitioners and the gynaecologists from our hospitals to send us first-catch urine (FCU) samples, from men and women, which were collected after the treatment of a C. trachomatis infection (the infection had been diagnosed earlier in our laboratories). Patients collected urine on the day they revisited their physician. The COBAS amplicor assay (Roche Molecular Diagnostics) was used to monitor the presence of $C$. trachomatis and thus the effectiveness of treatment.

One hundred and ten urine samples, one specimen per patient ( 35 male urine samples and 75 female urine samples), collected between 1 and 9 weeks after treatment, were tested with the COBAS amplicor assay. The results are shown in Fig. 1. Only seven of the 110 samples were positive during the entire observation period. Three urine samples were positive in the period 0-2 weeks after treatment. These samples were probably taken too soon after treatment. Four of the samples taken between 2 and 4 weeks after treatment were also positive. Although we can not completely exclude unsuccessful treatment, two of them were from patients with promiscuous conduct and one was from a patient with an untreated promiscuous partner, all suggesting reinfection. One sample (the only positive male urine, taken on day 18) had no clear indication of reinfection or ineffectiveness of treatment. Moreover, we could not completely rule out that the sample contained residual DNA from non-viable C. trachomatis organisms.

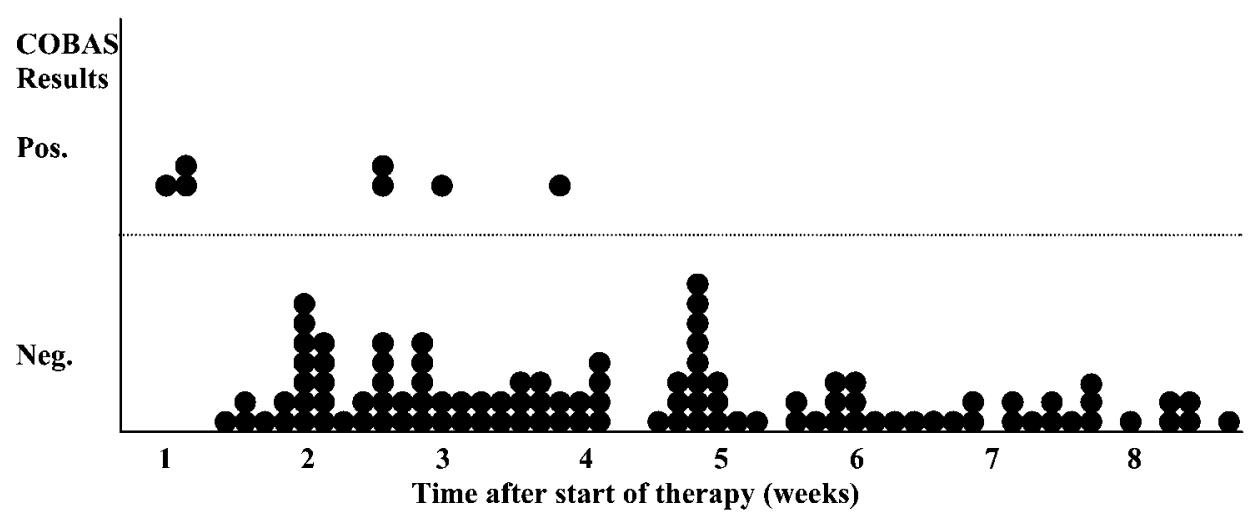

Fig. 1. Results of a nucleic acid amplification test for Chlamydia trachomatis on urine samples after treatment. Urine samples, collected after treatment of a C. trachomatis infection at the time points indicated, tested positive or negative in the COBAS amplicor assay. - First-catch urine sample. 
Such a sample might be negative in an assay for the detection of C. trachomatis RNA, as suggested by Morré et al. (1998).

In conclusion, our study on the monitoring of C. trachomatis infection showed that DNA detection in urine samples 2 weeks after treatment was negative, indicating successful treatment. One option for the few cases in which the sample remains positive 2 weeks after treatment would be to determine the presence of DNA again 4 weeks after treatment to exclude treatment failure or a reinfection with C. trachomatis.

\section{M. M. Salimans, ${ }^{1}$ C. Hol, ${ }^{1}$ M. C. A. Blans ${ }^{2}$ and A. G. M. van der Zanden ${ }^{2}$}

${ }^{1}$ Department of Medical Microbiology and Immunology, Meander Medical Centre, Amersfoort, The Netherlands

${ }^{2}$ Department of Medical Microbiology and Infectious Diseases, Gelre Hospitals, Apeldoorn, The Netherlands
Correspondence: M. M. M. Salimans (m.salimans@meandermc.nl)

Bianchi, A., Bogard, M., Cessot, G., Bohbot, J. M., Malkin, J. E. \& Alonso, J. M. (1998).

Kinetics of Chlamydia trachomatis clearance in patients with azithromycin, as assessed by first void urine testing by PCR and transcription-mediated amplification. Sex Transm Dis 25, 366-367.

Claas, H. C. J., Wagenvoort, J. H. T., Niesters, H. G. M., Tio, T. T., van Rijsoort-Vos, J. H. \& Quint, W. G. V. (1991). Diagnostic value of the polymerase chain reaction for Chlamydia detection as determined in a follow-up study. J Clin Microbiol 29, 42-45.

Gaydos, C. A., Theodore, M., Dalesio, N., Wood, B. J. \& Quinn, T. C. (2004). Comparison of three nucleic acid amplification tests for detection of Chlamydia trachomatis in urine specimens. J Clin Microbiol 42, 3041-3045.

Moncada, J., Chow, J. M. \& Schachter, J. (2003). Volume effect of sensitivity of nucleic acid amplification tests for detection of Chlamydia trachomatis in urine specimens from females. J Clin Microbiol 41, 4842-4843.
Morré, S. A., Sillekens, P. T. G., Jacobs, M. V. \& 7 other authors (1998). Monitoring of Chlamydia trachomatis infections after antibiotic treatment using RNA detection by nucleic acid sequence based amplification. J Clin Pathol 51, 149-154.

Roosendaal, R., Walboomers, J. M. M., Veltmann, O. R., Melgers, I., Burger, C., Blekers, O. P., MacClaren, D. M., Meijer, C. J. \& van den Brule, A. J. (1993). Comparison of different primer sets for detection of Chlamydia trachomatis by the polymerase chain reaction. J Med Microbiol 38, 426-433.

Van Doornum, G. J. J., Schouls, L. M., Pijl, A., Cairo, I., Buimer, M. \& Bruisten, S. (2001). Comparison between the LCx probe system and the COBAS AMPLICOR system for detection of Chlamydia trachomatis and Neisseria gonorrhoeae infections in patients attending a clinic for treatment of sexually transmitted diseases in Amsterdam, The Netherlands. J Clin Microbiol 39, 829-835.

Vogels, W. H. M., van Voorst Vader, P. C. \& Schröder, F. P. (1993). Chlamydia trachomatis infection in a high-risk population: comparison of polymerase chain reaction and cell culture for diagnosis and follow-up. J Clin Microbiol 31, 1103-1107. 